\title{
Expression systems
}

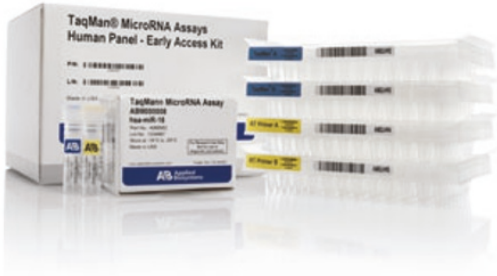

MicroRNA assays

TaqMan microRNA assays, from Applied Biosystems, are predesigned and validated probe and primer sets in a flexible format for the detection and quantification of mature human microRNA (miRNA) expression levels. The assays accommodate both large initial screening panels (many miRNAs in parallel) and in-depth further screening to determine differential expression levels of specific miRNAs. A set of 157 human assays with three nonvertebrate negative controls and an individually available miR-16 assay are currently available, with additional species and individual assays expected.

http://www.appliedbiosystems.com/

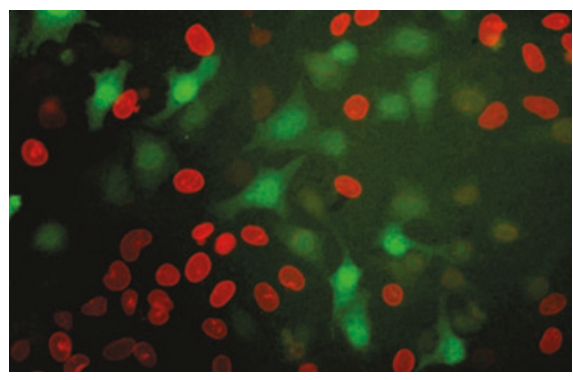

\section{Expression vector kits}

BLOCK-iT Pol II miR RNAi Expression Vector Kits from Invitrogen combine the high design success rate of artificial miRNAs and tissuespecific options of pol II promoters with the traditional advantages of RNA interference (RNAi) vectors. In addition to stable and/or regulated expression and viral delivery options, over $70 \%$ design success allows users to screen fewer clones. In addition, users can reliably track miRNAs with cocistronic GFP expression and easily knockdown various pathway components using multiple target knockdown sequences per transcript.

http://www.invitrogen.com/

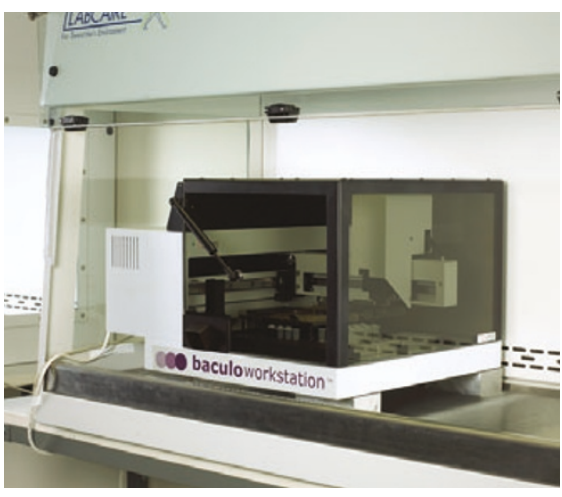

Baculovirus expression platform

NextGen Sciences' baculoworkstation is the first automated platform for robust, reproducible, high-throughput baculovirus protein expression. Designed to address the issues facing researchers using insect cells for protein expression, the baculoworkstation's core capabilities include the automation of cell seeding into 24well plates, transfections, infections, viral dilutions, and parallel expression screening. The system also has the facility for in-house protocol development, with application support provided by NextGen's development team.

http://www.nextgensciences.com/

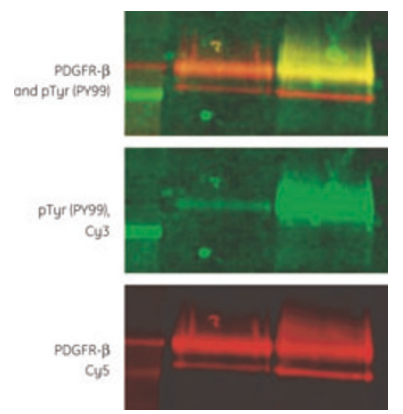

Western blot detection system

ECL Plex is a detection system that enables researchers to perform simultaneous multiwavelength protein expression analysis on western blots using Cy3 and Cy5 fluorescent dye labels, without the need for enzyme substrate amplification. It consists of products selected and optimized for best performance regarding sensitivity, dynamic range, linearity, and signal-to-noise ratio. In the multiplex application, two proteins can be detected with minimal cross-reactivity between antibodies and the long-lasting signal allows stored blots to be rescanned months later. The system is designed for existing multipurpose imagers such as Typhoon and Ettan DIGE.

http://www.gehealthcare.com/ 